\title{
Effect of amount of perceptual learning upon disappearances observed under reduced stimulation conditions ${ }^{\prime}$
}

\author{
RICHARD C. TEES AND LINDA K. MORE \\ UNIVERSITY OF BRITISH COLUMBIA
}

Perceptual learning was studied under reduced stimulation conditions. We found that, simply as a result of previous close temporal and spatial concurrence, discriminably different stimuli disappear together more frequently than without such association. Moreover, it was shown that the extent to which the stimuli subsequently "operated" together was a linear function of the frequency of the previous joint occurrence.

When an observer fixates on a luminous figure under conditions of low background illumination, parts of the target disappear and reappear (McKinney, 1963). Disappearance of visual stimuli during fixation at higher levels of illumination has also been observed (Craig \& Lichtenstein, 1953; Clarke, 1957, 1960). There appears to be a qualitative similarity in target fragmentation observed with partial retinal stabilization and this "stimulus induced" stabilization (Clarke \& Belcher, 1962; Evans \& Piggins, 1963; Pritchard, Heron, \& Hebb, 1960). In terms of central processes, McKinney (1963) and Donderi and Kane (1966) have proposed an explanation of the stimulus induced phenomena similar to Hebb's (1963) analysis of perceptual disappearances with restricted eye movements.

If disappearances under simplified stimulus conditions and retinal stabilization are both the result of central process inhibition, and if these neural processes develop as a result of perceptual learning (Hebb, 1949), then experience should influence subsequent disappearances. The problem is to employ a perceptual learning exercise on the basis of which predictions about later disappearances can be made. Because of its greater simplicity, Donderi and Kane (1965) used the reduced stimulation technique to study disappearance phenomena. They found that discriminably different stimuli, given a common response in a paired associate task or in stimulus recognition training, faded or disappeared together more frequently than if they had not been given the common response. The role response training plays in influencing perceptual disappearances has been questioned. Schuck and Leahy (1966) compared verbal and nonverbal reports of fragmentation and concluded that response training and bias did not affect the perception of stimuli but simply altered the reporting of their fragmentation.

The present study takes a different approach to the problem of a controlled perceptual learning experience. A modification of a method employed by Deitcher (1957), Tees (1960), and Melton (1963) for studying the nature of immediate memory was used. The purpose of this experiment was to examine the proposition that, simply as a result of previous close temporal and spatial concurrence, discriminably different stimuli disappear together more frequently under reduced stimulation conditions than without such association. Moreover, the hypothesis was also tested that the extent to which the stimuli subsequently disappeared together was a function of the frequency of their previous joint occurrence.

\section{Subjects}

\section{METHOD}

The Ss were 50 college student volunteers who were randomly assigned to five groups of 10 .

\section{Prelearning Test}

The stimuli were two luminous three-digit targets (855 and 332). The horizontally arranged digits were 3 in. high, 2 in. wide, $1 / 3$ in. thick, and equidistant from each other. At the 5-1/2 ft viewing distance these dimensions represented $2.7 \times 1.7 \times 0.3$ degrees. Horizontally, the three digits subtended an angle of 6.1 degrees. In a darkened, light-tight room, each $S$ viewed only one of the three-digit targets monocularly with the right eye for $5 \mathrm{~min}$. Five randomly assigned Ss from each of the five groups were exposed to the target 855 , the remaining five to 332 . The Ss were instructed to report only complete disappearances of digits.

\section{Percepfual Learning Experience}

The display materials were $2 \times 2$ in. slides projected on an $8-1 / 2 \times 11-1 / 2$ in. ground glass screen. Each slide contained from two to nine digits horizontally arranged and was automatically presented by a Kodak Carousel projector for $5 \mathrm{sec}$. With the $S$ sitting $2 \mathrm{ft}$ from the screen, each digit in the display was $2.8 \times 1.8$ degrees. Horizontally the digits in the slides subtended angles of 3.8-17 degrees. Under conditions of low background illumination, the $S$ fixated on the screen monocularly with the right eye and was asked to report the number of digits (two to nine) in each slide. Each $S$ was exposed to 100 slides. In these 100 slide presentations, the 
Table 1. Mean Frequency and Differences in Disappearances for

the Two Luminous Targets in the Five Exposure Conditions

\begin{tabular}{|c|c|c|c|c|c|c|c|c|}
\hline \multirow[b]{2}{*}{$\begin{array}{l}\text { Exposure } \\
\text { Frequency }\end{array}$} & \multirow[b]{2}{*}{$N$} & \multicolumn{6}{|c|}{ Target $A^{*}$} & \multirow[b]{2}{*}{$\begin{array}{c}\text { Mean Diff. } \\
\text { of Diff. } \\
\text { (1) }-(2)\end{array}$} \\
\hline & & $\begin{array}{l}\text { Before } \\
\text { Mean No. } \\
\text { Identical } \\
\text { Pairs }\end{array}$ & $\begin{array}{l}\text { Perceptual } \\
\text { Mean No. } \\
\text { Training } \\
\text { Pairs }\end{array}$ & $\begin{array}{l}\text { Learning } \\
\text { Mean } \\
\text { Difference } \\
\text { (1) }\end{array}$ & $\begin{array}{l}\text { After P } \\
\text { Mean No. } \\
\text { Identical } \\
\text { Pairs }\end{array}$ & $\begin{array}{c}\text { Merceptual } \\
\text { Mean No. } \\
\text { Training } \\
\text { Pairs }\end{array}$ & $\begin{array}{l}\text { Learning } \\
\text { Mean } \\
\text { Difference } \\
\text { (2) }\end{array}$ & \\
\hline 0 & 10 & 8.5 & 1.9 & 6.6 & 10.3 & 2.5 & 7.8 & -1.2 \\
\hline 30 & 10 & 8.5 & 2.3 & 6.2 & 9.9 & 4.7 & 5.2 & 1.0 \\
\hline 45 & 10 & 4.4 & 1.2 & 3.2 & 5.6 & 5.2 & .4 & 2.8 \\
\hline 60 & 10 & 6.3 & 1.0 & 5.3 & 2.2 & 4.8 & -2.6 & 7.9 \\
\hline 75 & 10 & 5.4 & 1.3 & 4.1 & 2.2 & 7.1 & -4.9 & 9.0 \\
\hline
\end{tabular}

${ }^{*}$ Target $A(e . g .855)$ seen in both pre-and post-learning tests.

training stimuli, the digit pairs 32 and 85 , were embedded $0,30,45,60$, and 75 times. In each of the above conditions, the number of occurrences of 32 was equal to the number of 85 pairings. For some presentations this resulted in both digit pairs (85 and 32) being embedded in the same slide. The crucial digits $(3,2,8$, and 5$)$ did not occur in any display except as a training pairing. One of the five groups of Ss was randomly assigned to each of the five stimulus exposure $(0,30,45,60$, and 75 pairings) conditions.

\section{Post-learning Test}

After the 100 slides, the $S$ returned to the prelearning test situation described above and the room was redarkened. All Ss viewed both three-digit targets on the post-learning test for $5 \mathrm{~min}$ each, and again were asked to report only complete digit disappearances. Which target was viewed first was a matter of random assignment.

\section{RESULTS}

The results concerning disappearances of the two targets (855 and 332) are presented in Tables 1 and 2. The reports of individual Ss were separated into disappearances related to "identical" or "training" stimulus pairs. Either the disappearance of the identical pair (e.g, -55) or the odd digit (e.g., 8--) is evidence for the effect of an association between identical stimuli on an individual's perception. The disappearance of the training pair (e.g., 85-) or the

Table 2. Mean Proportion of Training Pair (85 and 32) Disappearances for the Two Luminous Targets

\begin{tabular}{|c|c|c|c|c|c|c|}
\hline \multicolumn{3}{|c|}{$\begin{array}{c}\text { Before } \\
\text { Perceptual Learning } \\
\text { Target A }\end{array}$} & \multicolumn{4}{|c|}{$\begin{array}{c}\text { After } \\
\text { Perceptual Learning } \\
\text { Target A }\end{array}$} \\
\hline \multicolumn{3}{|c|}{$\begin{array}{l}\text { Exposure Mean SD } \\
\text { Frequency Proportion }\end{array}$} & $\begin{array}{l}\text { Mean } \\
\text { Propor }\end{array}$ & SD & $\begin{array}{l}\text { Mear } \\
\text { Propo }\end{array}$ & SD \\
\hline 0 & .120 & .170 & .168 & .114 & .153 & .176 \\
\hline 30 & .217 & .094 & .331 & .164 & .425 & .270 \\
\hline 45 & .176 & .181 & .468 & .240 & .569 & .346 \\
\hline 60 & 131 & .126 & .680 & .254 & .730 & .200 \\
\hline 75 & 159 & .187 & .804 & .148 & .808 & .189 \\
\hline
\end{tabular}

remaining digit (e.g.. --5) is evidence of an association between the training stimuli. Disappearances of all digits simultaneously cannot be attributed to a particular pair of digits and are not included in the analysis. The disappearance of the center digits (e.g., 8-5) and the two outside digits (e.g.,-5-) are also not reported. The center digit would be expected to disappear very frequently as a result of fixation; this, in fact, does occur. This influence of the point of fixation on disappearances has previously been reported (Hart, 1964; Tees \& More, 1966).

The proposition was tested that, before the perceptual learning exercise, disappearances related to identical digit pairs were more frequent than those related to the non-identical training pairs. For each of the exposure conditions, the exact binomial probabilities were found based on the hypothesis that the identical pairs did disappear as often as or less often than the non-identical (training) digit pairs did in the pre-learning tests. For 48 of the $50 \mathrm{Ss}$, the proportion of paired disappearances of identical targets was greater than 0.500 . Therefore, the hypothesis was rejected at $p<.0001$.

In order to demonstrate a significant perceptual learning effect, two post hoc tests were carried out on the mean difference between number of identical digit pair disappearances and number of training pair disappearances (see Table 1). Both the Tukey test and the Student Newman-Keuls test rejected the hypothesis of no difference between pre- and post-learning for the 60 and 75 exposure conditions $(p<.05)$.

To determine whether or not the amount of perceptual learning (as measured by the disappearance phenomena) varied with exposure frequency, a trend analysis of variance was carried out (see Table 3 ). Since the exposure frequencies were not equally spaced, the orthogonal polynomials (for the five mean differences of difference) were modified. The polynomials used for the linear trend analysis were -14 , $-4,1,6,11$. A highly significant linear trend was found $(F=33.62, \mathrm{df}=1 / 45, \mathrm{p}<.001)$ which accounted for $91.3 \%$ of the variance. No other trends were significant (see Fig. 1). 
Finally, inspection of the data presented in Table 2 indicates that the mean proportions of training pair disappearances for Targets $A$ and $B$ (after the perceptual learning experience) were comparable, thereby ruling out the possibility that the perceptual learning results were an artifact, produced by the pre-learning exposure to Target $\mathrm{A}$.

\section{Discussion}

In this experiment, the perceptual experience of a $\mathbf{S}$ was manipulated in order to assess the role sensory-sensory learning can play in the kind of luminous figure disappearances observed under simplified stimulus conditions.

First, however, comment is warranted concerning the perceptual learning exercise that has been employed. The exercise evolved from research cited earlier in this paper dealing with the nature of immediate memory. Ss were asked to attempt to repeat a series of digits, ostensibly to see whether practice would improve performance. Actually, every third (e.g.) set of digits was the same. Regardless of any general practice effect noted over trials, cumulative learning of the crucial series did take place. Reinforcement derived from being told of mistakes and correct series did not affect the rate of this learning (Tees, 1960). The important implications of this research with regard to learning, short-term memory, and central process changes has been discussed previously (Hebb, 1961; Melton, 1963).

If our Ss' response had been to repeat the digits observed in the visual display, several confounding variables would have been introduced. In the above procedure, reinforcement is not ruled out entirely. Though the Ss were not motivated to remember the crucial series of digits after the next had begun, they were certainly motivated to remember and repeat the individual sets of digits. Moreover, in repeating the series, the Ss derive an informational or feedback source of reinforcement. In the present experiment, having been asked to report the total number of digits in each visual display, the Ss were motivated to carry out a task irrelevant to the crucial perceptual learning experience. Thus engaged, it is less likely that their attention would be called to frequent concurrence of the digit pairs 32 and 85 in the targets. With the common-response and stimulus recognition tasks used by Donderi and Kane

Table 3. Trend Analysis of Difference of Differences for Five Exposure Conditions

\begin{tabular}{lcccc} 
Source of Variation & Sum of Squares & df & Mean Squares & $F$ \\
\hline Between Groups & 776.4 & 4 & 194.1 & \\
Linear Trend & 709.3 & 1 & 709.3 & $33.62 *$ \\
Other Trends & 67.1 & 3 & 22.4 & 1.10 \\
Within Groups (Error) & 948.1 & 45 & 21.1 & \\
Total & 1724.5 & 49 & & \\
\hline
\end{tabular}

$* p<.001$

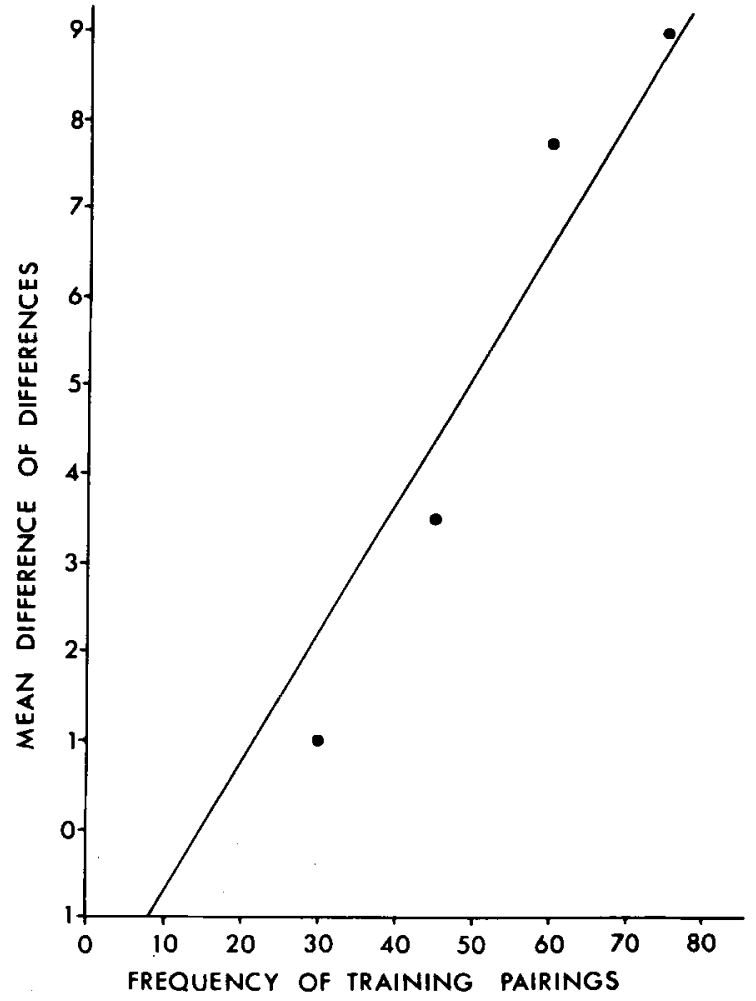

Fig. 1. Linear trend of mean differences of differences as a function of the number of preconditioning pairings (including best fitting regression line).

(1965) to produce controlled perceptual learning, there remains a greater possibility of confounding attentional and motivational variables.

However, the most important consideration regarding the problem of verbal reports has to do with the possible introduction of response training and/ or bias. If the $S$ simply repeats the digits in each visual display, not only are the visual stimuli 3 and 2 (as well as 8 and 5) appearing in close temporal and spatial connection but also these verbal responses are concurring with equal frequency. By having the $S$ report the total number of digits, the perceptual learning experience is restricted to $S-S$ associations rather than to response mediated associations that have been previously demonstrated (Donderi \& Kane, 1965; McKinney, 1966; Schuck \& Leahy, 1966).

With regard to the nature of the luminous target tests, it is important to recognize the consequences of the choice of digit triplets. Concurrent disappearance of parallel lines and identical forms have been reported when the visual image is stabilized on the retina (Replogle, 1962; Tees, 1961). Similar results have been obtained under reduced stimulation conditions (McKinney, 1963). Moreover, using forms scaled for similarity and simplified stimulus conditions, Donderi (1966) found the duration of simultaneous disappearance of two forms increased with 
an increase in their form-pair similarity. Under similar conditions two identical forms, digits or letters in a horizontally-arranged three-element display, disappear more frequently together than any other pair (Tees \& More, 1966). In this investigation, the perceptual learning experience worked against the fact that identical stimuli disappear together more frequently than discriminably different stimuli. Even though 85 and 32 were selected as the test pairs, the effects of their previous association in the perceptual learning situation significantly influenced the form disappearances took in the postlearning test. Simply as a product of 60 previous pairings a perceptual association was established between two discriminably different stimuli. A theoretical basis for perceptual associations has been described previously by Hebb (1949). The outcome of this experiment seems to concur with Hebb's original hypothesis concerning the development of cell assemblies and phase sequences solely as a result of contiguity. As a consequence of their previous close temporal and spatial concurrence, the neural representatives of the stimuli reliably inhibit and facilitate each other's firing, and this is evidenced in joint disappearances in the post-learning test. Moreover, there appears to be little way in which common response training or blas could have been involved in the development of this perceptual association.

The fact that as many as 60 paired exposures may be required to produce significant evidence of perceptual learning is not surprising. The nature of the S's task (counting the number of digits in each display) certainly results in lack of attention to the cruclal pair, digits per se, and perhaps even the entire task itself. The targets, as seen on the ground glass screen, were not made up of perfectly-formed, sharply contrasted digits, and it can be well imagined that the task might reduce itself to counting shapeless forms in each display.

Inspection of Fig. 1 as well as the results of the linear trend analysis suggest the effect of increasing the number of learning experiences on integration of the neural representatives of the training pair is gradual and linear. Inspection of Table 2 reveals another interesting finding. A second target was viewed solely in the post-learning situation in order to check whether experience in the pre-learning test with digit triplets did itself influence later disappearances in the post-test. If the proportions of training pair disappearances with the target seen solely in the post-test had been significantly lower than those obtained with the training pair seen in both pre- and post-learning tests, data interpretation involving attentional variables might be suggested. This was not so. In fact, evidence of perceptual learning with this target was consistently greater for each exposure frequency. In general, perceptual disappearances under reduced stimulation conditions seem to provide a remarkably sensitive index of the strength of perceptual associations. However, the collection of disappearance duration information may provide an even better measuring device.

\section{References}

Craig, E. A., \& Lichtenstein, M. Visability-invisability cycles as a function of stimulus orientation. Amer. J. Psychol., 1953, $66,554-563$.

Clarke, F. J. Rapid adoption of localized areas of the extra-foveal retina. Optica Acta, 1957, 4, 69-77.

Clarke, F. J. A study of Troxler's effect. Optica Acta, 1960, 7 , 219-236.

Clarke, F. J., \& Belcher, S. J. On the localization of Troxler's effect in the visual pathway. Vis. Res., 1962, 2, 53-68.

Deitcher, J. Memory trace in digit repetition. Unpublished Honor thesis, McGill University, 1957.

Donderi, D. C. Visual disappearances casued by form similarity. Science, 1966, 152, 99-100.

Donderi, D. C., \& Kane, E. Perceptual learning produced by common responses to different stimuli. Canad. J. Psychol., 1965, $19,15-30$.

Edwards, A. L. Experimental design in psychological research. New York: Holt, Rinehart \& Winston, 1960.

Evans, C. R., \& Piggins, D. J. A comparison of the behaviour of geometrical shapes when viewed under conditions of steady fixation and with apparatus for producing a stabilized retinal image. Brit. J. physiol. Opt, 1963, 20, 1-13.

Hart, J. T. Luminous figures: influence of point of fixation on their disappearance. Science, 1964, 143, 1193-1194.

Hebb, D. O. The organization of behaviour. New York: Wiley, 1949.

Hebb, D. O. Distinctive features of learning in the higher animal. In J. F. Delafresnaye (Ed.), Brain mechanisms and learning. Springfield: Thomas, 1961. Pp. 37-51.

Hebb, D. O. The semiantonomous process: its nature and nurture. Amer. Psychol., 1963, 1, 16-27.

McKinney, J. P. Disappearance of luminous designs. Science, $1963,140,403-404$.

McKinney, J. P. Verbal meaning and perceptual stability. Canad. J. Psychol., 1966, 20, 237-242.

Melton, A.W. Implications of short-term memory for a general theory of memory. J. verbal Learn, verbal Behav., 1963, 2, 1-21.

Pritchard, R. M., Heron, W., \& Hebb, D. O. Visual perception approached by the method of stabilized images. Canad. J. Psychol., 1960, 14, 67-77.

Replogle, A. The effects of similarity on the behaviour of perceived figures. Paper read at EPA, Atlantic City, 1962.

Schuck, J. R., \& Leahy, W. R. A comparison of verbal and non-verbal reports of fragmenting visual images. Percept. \& Psychophys., 1966, 1, 191-192.

Tees, R. C. The effects of reinforcement on digit repetition. Underg. res. proj. Psychol., 1960, 2, 64-70, (McGill University).

Tees, R. C. The role of field effects in visual perception. Underg. res. proj. Psychol., 1961, 3, 87-96, (McGill University).

Tees, R. C., \& More, L. K. Identical forms, exposure time and disappearance phenomena under reduced stimulation conditions. Psychon. Sci., 1966, 6, 289-290.

\section{Nofe}

1. This research was supported with funds granted by the National Research Council of Canada (Grant APA-179) and by the University of British Columbia Faculty of Graduate Studies' Committee on Research. The authors gratefully acknowledge the assistance of Terence D. Creighton.

(Accepted for publication August 4, 1967.) 